\section{Updated Diagnosis and Management of Osteoarticular Tuberculosis}

\author{
Shou-Chien Chen ${ }^{1,2}$ and Kow-Tong Chen ${ }^{3,4 *}$ \\ ${ }^{1}$ Department of Family Medicine, Da-Chien General Hospital, Miaoli, \\ Taiwan \\ ${ }^{2}$ General Education Center, Ta Tung University, Taipei, Taiwan \\ ${ }^{3}$ Department of Occupational Medicine, Tainan Municipal Hospital, Tainan, \\ Taiwan
}

${ }^{4}$ Department of Public Health, College of Medicine, National Cheng Kung University, Tainan, Taiwan

\begin{abstract}
\section{Background}

Tuberculosis (TB) of the osteoarticular system usually manifests as osteomyelitis and joint arthritis. The diagnosis of TB arthritis is often delayed due to the lack of awareness, insidious onset, lack of characteristic early radiographic findings and, often, lack of constitutional or pulmonary involvement. The purpose of this review is to provide an update on the diagnostic and therapeutic recommendations for TB arthritis.

Methods

The literature from a range of sources was reviewed and synthesized to provide an overview of contemporary approaches for the diagnosis and management of TB arthritis.

\section{Results}

This review summarizes the clinical, radiological, bacteriological, immunological, synovial fluid examination, and synovial biopsy approaches used in the diagnosis of TB arthritis. We also summarize the updated guideline for the treatment of TB arthritis.

\section{Conclusion}

Intense current and previous efforts into diagnostic, therapeutic, and preventive interventions have focused on pulmonary TB in adults, but TB arthritis has been relatively neglected. The development of better diagnostic and therapeutic methods for TB arthritis remains a significant challenge. As the strategies for the diagnosis and management of TB arthritis improve and the knowledge base increases, the implementation of these strategies will be crucial.
\end{abstract}

*Corresponding author: Kow-Tong Chen, Department of Occupational Medicine, Tainan Municipal Hospital, No. 670, Chongde Road, East District, Tainan, Taiwan, Tel: 88662609926; Fax: 88662606351; E-mail: kowton@ms81.hinet.net; ktchen@mail.ncku.edu.tw

Citation: Chen SC, Chen KT (2014) Updated Diagnosis and Management of Osteoarticular Tuberculosis. J Emerg Med Trauma Surg Care 1: 002.

Received: September 07, 2014; Accepted: November 27, 2014; Published: December 11, 2014

\author{
Keywords: Diagnosis; Management; Tuberculosis arthritis; \\ Tuberculosis
}

\section{Introduction}

Tuberculosis (TB) remains a major health problem worldwide. In 2011, the World Health Organization (WHO) reported an estimated 8.7 million incident cases of TB, 12 million prevalent cases of TB, and 1.4 million deaths due to TB [1]. Most cases occurred in Asia (59\%) and Africa (26\%) [1,2]. As for active clinical TB, the most common form of tubercular disease is Pulmonary TB (PTB); nevertheless, in a widely variable proportion of cases, TB can also affect extra-pulmonary sites within the body, exclusive of or in combination with PTB [3].

Musculoskeletal tuberculosis is a relatively rare extra-pulmonary complication of Mycobacterium tuberculosis. Specific skeletal involvement is observed in $1 \%$ to $3 \%$ of patients with TB [4]. Among them, approximately one-half of cases show spinal involvement, and the remaining involve the extraspinal osteoarticular joint [5-7]. TB tenosynovitis and arthritis is usually monoarticular, and the organism can be isolated from the joint [8].

TB tenosynovitis and arthritis occurs primarily through hematogenous spread from a primary focus such as the lung, kidney, or lymph node or, infrequently, through contiguous spread from adjacent tissues by direct inoculation [8-12]. TB of the joint may be due direct invasion of the synovium, e.g., Poncet's arthritis $[8,11]$. It may also affect nonweight-bearing joints such as the wrist, elbow, and the small joints of the hands. Articular disease often starts as a synovitis progressing to periarticular demineralization, marginal erosions, and lastly, joint destruction [13]. The time period from synovitis to joint destruction can be rapid, particularly in weight-bearing joints. When tubercular tenosynovitis and arthritis are complicated by a secondary infection such as Staphylococcus aureus presents, they result in accelerated joint destruction associated with severe systemic features [14]. Joint tissue necrosis secondary to other diseases, as in the cases of osteonecrosis of the joints due to sickle cell disease and chondrocalcinosis may predispose an individual to tubercular infection $[15,16]$.

Patients generally have mild local and constitutional symptoms, frequently leading to significant delays in diagnosis. The diagnosis of tubercular tenosynovitis and arthritis is also frequently delayed due to its varied clinical presentation and frequent lack of radiographic findings and constitutional features [7,17]. The delay in diagnosis and treatment, may result in additional bone or joint destruction, particularly in patients with TB tenosynovitis and arthritis due to infection caused by mycobacterial species $[7,18]$. Therefore, early diagnosis and treatment are essential. We therefore performed a review of these topics based on the recent literatures.

\section{Data Collection}

We initially collected all of the articles that were published from January 1990 through March 2014, which described subjects affected by TB tenosynovitis and arthritis. These articles were obtained by searching MEDLINE (National Library of Medicine, Bethesda, 
Maryland, USA) using the key words "osteoarticular tuberculosis" [OR] "tuberculosis arthritis" [OR] "diagnosis of TB arthritis" [OR] "management of TB arthritis". All of the articles are included. Manuscripts without an abstract (which were assumed to not be original), and opinion articles were excluded from the review. After selecting the articles, the relevant information was extracted and classified according to TB arthritis diagnosis, TB arthritis management, the country of study, and the study design.

Literature searches were performed in July 2014 and August 2014 Using the search terms previously described, a total of 161 documents were retrieved from MEDLINE. After screening the articles, a total of 103 articles were considered to be relevant. The countries were primarily from India, the United States, Turkey, Korea, Taiwan, Germany and other countries. The setting that produced the most original information was India, with $35 \%$ of the articles. After analyzing the abstracts, we found that $83 \%$ of the studies were case reports, $10 \%$ were retrospective, and $7 \%$ referenced other designs.

\section{Diagnosis of TB Infection}

\section{Tuberculin skin test}

Table 1 shows the methods to diagnose tuberculosis arthritis. The Mantoux test is the recommended standard Tuberculin Skin Test (TST). Tuberculin is commercially available in 1, 2, and 5 Tuberculin Unit (TU) PPD (Purified Protein Derivative, RT23 equivalent) forms $[19,20]$. For the test, it is important to raise a wheal of approximately $6 \mathrm{~mm}$ after the intra-dermal injection. The test is read 48-72 hours after an injection. Ballpoint or palpatory methods are used to read the induration. The influence of a prior Bacillus Calmette-Guérin (BCG) vaccine on the $\mathrm{PPD}$ reaction depends on conditions such as the interval between the BCG vaccination and the TST and the age at vaccination [21]. If the prevalence of TB infection is high enough, the positive predictive value of the TST is also high [22]. If the patient returns for a reading beyond 72 hours but before the $7^{\text {th }}$ post-injection day, a positive test can still be read [23]. A repeat test may be needed if there is no induration and the wheals present beyond the stipulated time for reading. A repeat tuberculin test, when required, should preferably be performed on the other arm. Although the TST is widely used for diagnosing TB infection, it has some limitations. It is reported that the sensitivity may be decreased due to some factors like malnutrition, severe tuberculosis disease and immunodeficiency. In addition, the biggest disadvantage of TST is the cross-reaction with nontuberculous mycobacteria or with Mycobacterium bovis vaccine strains [24].

\begin{tabular}{|l|}
\hline \multicolumn{1}{|c|}{ Methods } \\
\hline Tuberculin Skin Test (TST) \\
\hline Interferon Gamma Release Assays (IGRAs) \\
\hline Enzyme-Linked Immunosorbent Assays (ELISA) \\
\hline Bacteriology \\
\hline Radiology \\
\hline Computed Tomography (CT) \\
\hline Magnetic Resonance Imaging (MRI) \\
\hline Polymerase Chain Reaction (PCR) \\
\hline Synovial fluid examination \\
\hline Synovial biopsy \\
\hline
\end{tabular}

Table 1: Summarizing the methods to diagnose tuberculosis arthritis.

\section{Interferon Gamma Release Assays (IGRAs)}

In addition to the traditional TST, which is known to lack both sensitivity and specificity, blood-based assays have recently become available. These T-cell assays rely on the stimulation of host blood cells with $M$. tuberculosis-specific antigens and measure the production of Interferon (IFN)- $\gamma$. Several studies have compared the two available commercial assays, T-Spot TB (Oxford Immunotec, UK) and QuantiFERON-TB Gold (Cellestis, Australia), with the TST for both the detection of active disease and Latent Tuberculosis Infection (LTBI) [25]. The T-cell assays have proven to be more specific than the TST, but they are currently unable to distinguish between active disease and LTBI [25]. Therefore, interpretation of the results remains dependent on the clinical context. Two new IGRAs may offer improved specificity and sensitivity over the TST for the diagnosis of LTBI $[26,27]$. One of these, QuantiFERONTB Gold (QFT-Gold), showed encouraging results in low-risk BCG-vaccinated subjects [28] and patients with active TB [27]. QFT-Gold overcomes some of the shortcomings of the TST, such as the need for return visits, reader variability, variable specificity, and cross reactivity with BCG vaccination and nontuberculous mycobacterial infections $[21,29,30]$. Although these tests are valuable in screening for LTBI, the diagnostic accuracy varies according to the patient population. Overall, the results indicate that IGRAs have a modest predictive value similar to that of the TST. In settings with a low $\mathrm{TB}$ incidence, the IGRAs demonstrated a higher specificity (100\% and $98 \%$ for the QuantiFERON-TB assay and the T-Spot assay, respectively) than the TST $(58 \%)$ in patients with TB $[31,32]$. The results from a meta-analysis, the pooled sensitivity was $78 \%(95 \%$ CI, 73-82\%) for QuantiFERON-TB Gold, 70\% (CI, 63-78\%) for QuantiFERON-TB Gold In-Tube, and 90\% (CI, 86\% to 93\%) for T-SPOT.TB; the pooled specificity for QuantiFERON tests and T-SPOT.TB is $99 \%$ (CI, 98-100\%) and 93\% (CI, 86-100\%), respectively, among non-BCG vaccinated subjects, but pooled specificity for TST in non-BCG vaccinated subjects is $97 \%$ (CI, 95-99\%) [33]. The costs and technical demands of IGRAs will most likely limit their wider use in resource-poor settings, where better tests are the most needed.

In the general population, IGRAs appear to be more powerful than the TST for diagnosing active TB or LTBI [34]. T-SPOT, QuantiFERON-TB and the TST have good diagnostic value for chronic inflammatory arthritis, but indeterminate results may complicate their use [35].

\section{Enzyme-Linked Immunosorbent Assays (ELISA)}

Antibody detection tests (serological tests), used for the diagnosis of several infectious diseases, may potentially improve TB diagnosis. These tests measure the presence of specific antibodies directed against immunodominant antigens of the investigated pathogen. Compared to smear microscopy, antibody detection methods may enable rapid TB diagnosis, as these tests have the advantages of being quick (results can be available within hours) and technologically easy, requiring minimal training. In addition, these tests could be adapted to point-of-care formats that can be implemented at lower levels of health services in low- and middleincome countries [36,37]. The serology tests used for diagnosing TB have a long record in the TB literature, but have never been well-developed, due to their low diagnostic values with poor specificity and sensitivity [38]. Since the 1990s, newer approaches have been chosen using ELISA and highly purified 
antigens or recombinant proteins [37]. Improvement of their performances has been obtained by using several different antigens simultaneously [39]. Serological tests based on ELISA using a panel of non-protein antigens such as glycolipids specific for $M$. tuberculosis have been developed and evaluated [40].

\section{Diagnosis of tuberculosis tenosynovitis and arthritis}

Clinical presentation: TB arthritis commonly presents with chronic joint pain and only minimal signs of inflammation. Tubercular arthritis is characteristically monoarticular and most commonly affects the spine and weight-bearing joints such as the knee, hip, and ankle; the synovial type of TB arthritis is more commonly involved in the knee, hip, and ankle joint [12].

The most common symptom is local pain and swelling followed by restriction of movement of affected area. There is wasting of the regional muscle, and deformity may occur. Less commonly, a painless cold abscess has been reported as the only clinical presentation. The involvement of multiple sites is observed in 5-30\% cases of tubercular arthritis $[41,42]$. Reactivation of tubercular arthritis after treatment occurs in $17-34 \%$ of individuals and is most commonly observed in the hip joint $[8,43]$.

Joint swelling and evidence of effusion, periarticular abscess and chronic sinus formation occur late. Systemic symptoms of fever, weight loss, and night sweats may or may not be present during active TB tenosynovitis and arthritis. Less than 50\% of individuals with tubercular tenosynovitis and arthritis have active pulmonary $\mathrm{TB}$ at the time of diagnosis [44]. Patients with TB may have hypersensitivity phenomena such as erythema nodosum, episcleritis, uveitis and Poncet's arthritis.

Clinically, TB tenosynovitis and arthritis has been classified into 5 stages [45-47]. Stage I, or the synovitis stage, presents with soft tissue swelling, no bony lesions, and localized osteoporosis, and the outcome after treatment is excellent. Stage II is early arthritis with marginal erosions (one or more erosions or lytic lesions in the bone; discrete diminution of the joint space), and the outcome is good, with only mild stiffness [6]. Stage III is advanced arthritis with subperichondrial cyst formation and loss of joint space; the outcome is fair, with notable loss of motion [6]. Stage IV is more advanced arthritis with joint destruction and no motion at the joint after treatment. Stage V is ankylosis of the joint.

Bacteriology: A confirmation of Acid Fast Bacillus (AFB) from any body fluid or tissue is the gold standard for the diagnosis of tuberculosis. Several studies have reported bacteriological positivity rates as high as $33 \%$ even for primary disease states, such as hilar adenopathy [48]. Therefore, every attempt must be made to bacteriologically prove the diagnosis in every case of suspected tuberculosis. Whatever method a clinician uses, he/she needs to collect at least two, preferably three, samples. A Ziehl-Neelsen (ZN) stain can reveal AFB only if the sample contains greater than 10,000 bacilli per $\mathrm{mL}$. Different culture methods, such as Lowenstein-Jensen medium and radiometric (Bactec 12B liquid medium) and non-radiometric (Bactec MGIT 960 system) culture, can be used to confirm the diagnosis in the paucibacillary state [49]. The newer methods are capable of providing faster results and may be used if available. Mycobacterial culture assumes special significance in cases of suspected drug resistance [49].

There is a push to switch from ( $\mathrm{ZN}$ to auramine microscopy. Despite World Health Organization guidelines that one staining method is sufficient, in some countries national guidelines prescribe that auramine-positive samples should be confirmed by ZN [50]. A positive auramine result followed by a negative $\mathrm{ZN}$ result could not be used to exclude TB or to indicate the presence of NTM species. Confirming auramine-positive samples using $\mathrm{ZN}$ provided no clinically informative information and was a waste of resources.

Based on the results of bacteriological methods, Multidrug Resistant (MDR) tuberculosis is defined as tuberculosis resistant to at least both isonizid and rifampicin [51]. Extensively Drug-Resistant (XDR) tuberculosis is defined as tuberculosis resistant to isoniazid and refampicin; and any fluoroquinolone; and at least one of the three second-line injectable drug (capreomycin, kanamycin, and amikacin) [51]. Recently, WHO has revised the case definitions of TB and drug-resistant TB so that also the results of WHO-endorsed new molecular and nonmolecular methods can be used to define a case. Case definitions were further revised to avoid judgmental language, so the terms "defaulter" and "TB suspect" have been replaced by "lost to follow-up" and "presumptive TB", respectively [51].

The main Drug Susceptibility Testing (DST) methods are the absolute-concentration method and the Proportion Method (PM) on Lowenstein-Jenson (L-J) medium, but both methods take some weeks for the results. Automation of culture using BACTEC MGIT 960 (M960) system is being widely implemented [52].

Accurate and rapid detection of Tuberculosis (TB) and drug resistance are critical for improving patient care and decreasing the spread of TB. The Xpert MTB/RIF assay, which enables simultaneous detection of Mycobacterium Tuberculosis (MTB) and Rifampicin (RIF) resistance, was endorsed by WHO in 2010 [53]. Xpert may also be valuable as an add-on test following microscopy for patients who have previously been found to be smear-negative. An Xpert result that is positive for rifampicin resistance should be carefully interpreted and take into consideration the risk of MDR-TB in a given patient and the expected prevalence of MDR-TB in a given setting.

Radiology: Radiographic features are usually noted 2 to 5 months after disease onset [7]. The classical triad of radiologic characteristics of TB tenosynovitis and arthritis is juxta-articular osteoporosis, peripheral osseous erosion and gradual narrowing of the intra-articular space (Figure 1) $[6,54,55]$. In contrast to rheumatoid arthritis, the joint space is relatively preserved in early $\mathrm{TB}$ arthritis. In children, an enlargement of the epiphysis may be observed. A bone scan shows increased uptake, but bone scan findings are non-pathognomonic.

Imaging: Computed Tomography (CT) and Magnetic Resonance Imaging (MRI) are helpful in further defining the disease (Figure 2) $[6,56]$. MRI defines soft tissues better, while CT is good for bony lesions. The MRI features of tubercular tenosynovitis and arthritis include synovitis, effusion, central and peripheral erosions, active and chronic pannus, abscess, bone chips, and hypo-intense synovium. MRI is the investigation of choice to reveal both the extent and severity of damage [16]. MRI is also nonspecific but better depicts the extent of the lesion when compared with X-rays. These imaging features in an appropriate clinical setting may aid the diagnosis of tubercular tenosynovitis and arthritis [55]. Positron Emission Tomography (PET) using 2-[18F]-Fluoro-2-Deoxy-D-Glucose (FDG) has been used to diagnose in breast, colorectal, head and neck, lung, lymphoma, melanoma, oesophageal and thyroid cancers [57]. However, other management decisions require further research to 


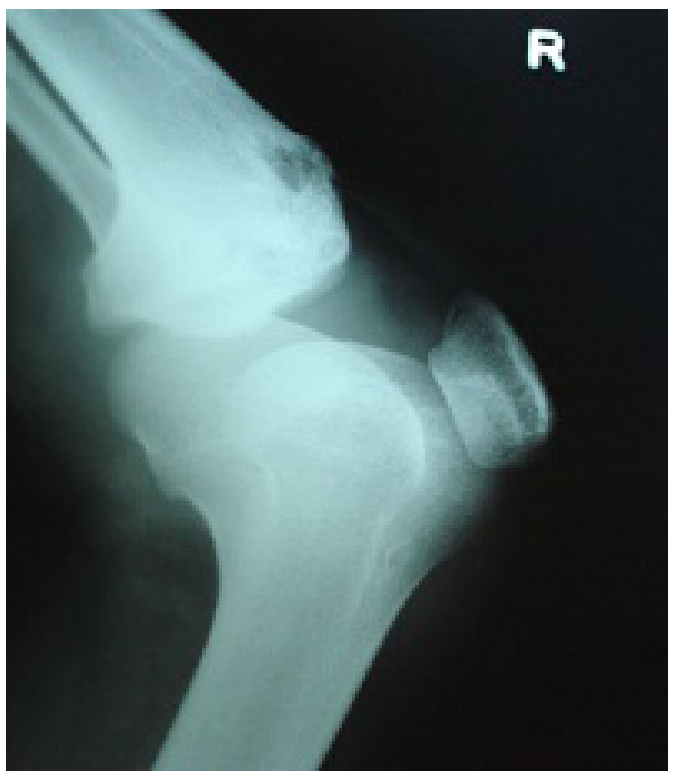

Figure 1: Radiograph of tuberculosis tenosynovitis and arthritis at tibial tuberosity. Swelling was present with or without a draining sinus. Radiographs showed erosion or lucent lesion at the site of the tendon attachment to bone [6].

show the impact of FDG-PET on patient management or added value in the diagnostic pathway for tuberculosis arthritis.

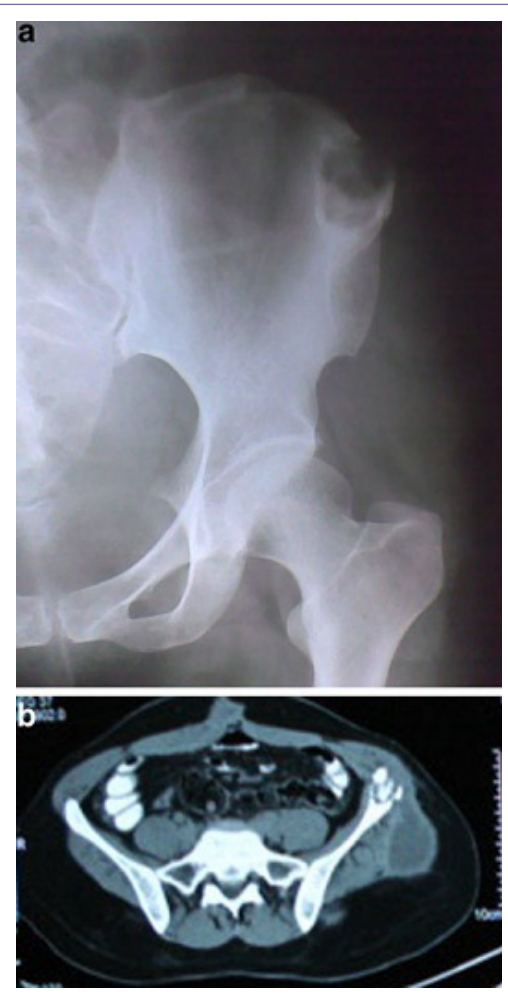

Figure 2: Comparison of imaging of radiography and Computed Tomography (CT). (a) The sequestrum is not apparent on the plain radiograph. (b) $A$ tubercular sequestrum in the left iliac crest are noted on CT imaging [6].

PCR testing: Nucleic acid amplification tests using Polymerase Chain Reaction (PCR) cannot differentiate living bacilli from dead bacilli. Thus, these tests continue to give positive results even after successful treatment. PCR is positive in $95 \%$ to $100 \%$ of culture-positive cases and in $50 \%$ to $60 \%$ of culture-negative cases [58]. In one study, a multiplex real-time PCR with species-specific primers based on senX3-regX3+IS711 sequences proved to be sensitive and specific and useful for the highly efficient detection of $M$. tuberculosis and Brucella spp. in different clinical samples [59]. Over the past several decades, the diagnostic methods for M. tuberculosis have improved, and nucleic acid amplification techniques now allow rapid and sensitive detection in clinical settings [60].

Synovial fluid examination: Synovial fluid is usually nonhemorrhagic and turbid, with moderate elevation of the white blood cell count, ranging between 10,000 and 20,000 cells $/ \mathrm{mL}$ with a predominance of polymorphonuclear leukocytes. Culture for $M$. tuberculosis should also be planned. Joint fluid aspiration from the affected joint for standard/routine investigation and TB culture is recommended, when possible, for at-risk patients, even if previous cultures have been negative. Synovial fluid culture is positive in approximately $20-40 \%$ of cases $[61,62]$. PCR analysis in synovial fluid, tissue samples, bone marrow aspirate, and peripheral blood is faster and more specific but less sensitive and less widely available $[62,63]$.

Synovial biopsy: The gold standard for the diagnosis of tubercular arthritis is synovial biopsy, with positive results in $80 \%$ of cases [64]. It shows caseating granulomas, lymphocyte infiltration, and giant cells with caseation, which are very characteristic of tubercular arthritis.

\section{Management of TB arthritis}

Splints may be used for a short time to relieve acute symptoms and for a long time in specific cases of tuberculosis of the joints to prevent deformities of the infected extremities [65,66]. Operative treatment is usually limited and includes obtaining a biopsy and performing open or arthroscopic debridement, incision and drainage of abscesses, and synovectomy [65,67]. A randomized trial performed primarily among ambulatory patients by the Medical Research Council Working Party on Tuberculosis of the Spine [68] demonstrated no additional benefit of surgical debridement or radical operation (resection of the spinal focus and bone grafting) in combination with chemotherapy when compared with chemotherapy alone. In some circumstances, however, surgery appears to be beneficial and may be indicated. Such situations include failure to respond to chemotherapy with evidence of ongoing infection, the relief of cord compression in patients with persistent of recurrence of neurological deficits, or instability of the spine. Surgical procedures should be restricted to joints with severe cartilage destruction, joint deformity, large abscesses, multiple drug resistance $[66,68,69]$.

The mainstay treatment of TB arthritis is appropriate anti-TB drug therapy (Table 2). Early antimicrobial therapy can result in near-complete resolution and preservation of function. In TB arthritis without pulmonary involvement, the risk of transmission to contacts is minimal and thus constitutes little threat to public health. Antimicrobial therapy in general should be of at least 6-9 months duration but should be continued longer in children and immunocompromised hosts $[9,12,13]$. The basic principles that underlie the treatment of pulmonary tuberculosis also apply to extra-pulmonary forms of the disease (Table 2) [54]. Although many fewer studies have examined the treatment of extra-pulmonary tuberculosis compared with pulmonary disease, increasing evidence, including some randomized controlled trials, suggests that the 6- to 9-month regimens (2 months of Isoniazid (INH) and Rifampicin (RIF), Pyrazinamide (PZA), and Ethambutol (EMB) followed by 4-7 months of INH and RIF) are recommended as initial therapy unless 
the organisms are known to be or strongly suspected of being resistant to the first-line drugs. If PZA cannot be used in the initial phase, the continuation phase must be increased to 7 months, as described for pulmonary tuberculosis. Several studies have examined the treatment of bone and joint tuberculosis and have shown that the 6-9-month regimens containing RIF are at least as effective as the 18-month regimens that do not contain RIF [68,70,71]. Myelopathy with or without functional impairment most often responds to chemotherapy. In two Medical Research Council studies conducted in Korea, 24 of 30 patients in one study [70] and 74 of 85 patients in an earlier study [72] had complete resolution of myelopathy or complete functional recovery when treated medically. The recommended dosages are listed in table $3[23,54]$.

\begin{tabular}{|c|c|c|c|}
\hline \multirow{2}{*}{$\begin{array}{c}\text { Category of } \\
\text { treatment }\end{array}$} & \multirow[b]{2}{*}{ Category of TB cases } & \multicolumn{2}{|c|}{ Anti-TB drug regimens } \\
\hline & & $\begin{array}{l}\text { Intensive } \\
\text { phase }\end{array}$ & $\begin{array}{c}\text { Continuation } \\
\text { phase }\end{array}$ \\
\hline 1 & $\begin{array}{l}\text { New Patient Regimen } \\
\text { New smear-positive PTB } \\
\text { Smear-negative PTB with } \\
\text { extensive } \\
\text { Parenchymal involvement } \\
\text { Severe forms of EPTB other } \\
\text { than TB meningitis }\end{array}$ & 2HRZE & $4 \mathrm{HR}$ \\
\hline ॥ & $\begin{array}{l}\text { New Patient Regimen } \\
\text { Smear-negative PTB without } \\
\text { extensive } \\
\text { P Parenchymal involvement } \\
\text { Less severe forms of EPTB } \\
\text { (e.g., TB cervical adenitis) }\end{array}$ & $2 \mathrm{HRZ}$ & $4 \mathrm{HR}$ \\
\hline III & $\begin{array}{l}\text { New Patient Regimen } \\
\text { TB meningitis }\end{array}$ & $2 \mathrm{HRZS}^{\mathrm{a}}$ & $4 \mathrm{HR}$ \\
\hline IV & $\begin{array}{l}\text { Retreatment regimen } \\
\text { Previously treated } \\
\text { smear-positive PTB (relapse, } \\
\text { treatment after } \\
\text { interruption or treatment } \\
\text { failure) } \\
\text { If low risk for MDR-TB or risk } \\
\text { unknown, continue with } \\
\text { retreatment regimen } \\
\text { If high risk for MDR-TB, use } \\
\text { MDR-TB regimen below }\end{array}$ & $\begin{array}{c}2 \mathrm{HRZ}- \\
\mathrm{ES} / 1 \mathrm{HRZE}\end{array}$ & 5HRE \\
\hline $\mathrm{v}$ & $\begin{array}{l}\text { MDR Regimen } \\
\text { MDR-TB }\end{array}$ & $\begin{array}{l}\text { Individualized } \\
\text { regimens }\end{array}$ & \\
\hline
\end{tabular}

Table 2: Treatment regimens for tuberculosis recommended by the WHO [42].

a Other regimens are recommended for the treatment of TB meningitis, including replacing streptomycin with ethionamide and treating for 9-12 months.

E: Ethambutol; EPTB: Extra-Pulmonary Tuberculosis; H: Isoniazid; HIV: Human Immunodeficiency Virus; MDR-TB: Multi-Drug Resistant Tuberculosis; PTB: Pulmonary Tuberculosis; R: Rifampicin; S: Streptomycin; Z: Pyrazinamide; 2HRZ 4HR: Denotes a Two-month Intensive Phase of Daily Isoniazid, Rifampicin, and Pyrazinamide followed by a Four-month Continuation Phase of Daily Isoniazid and Rifampicin

\section{Conclusions}

TB is still an important public health problem throughout the world. TB arthritis accounts for approximately 1-3\% of all cases of tuberculosis and for approximately $10-11 \%$ of extra-pulmonary cases. Nonspinal skeletal TB is rare and insidious in onset and is often difficult to diagnose. Early diagnosis and specific and adequate treatment can improve the possibility of maintaining good joint function.

\begin{tabular}{|c|c|c|}
\hline & Recommended & Dose per day \\
\hline Drug & Adults & Children \\
\hline Isoniazid & $\begin{array}{c}\text { Dose and range } \\
\text { (mg/kg body weight) }\end{array}$ & $\begin{array}{c}\text { Dose and range } \\
\text { (mg/kg body weight) }\end{array}$ \\
\hline Rifampicin & $5(4-6)$ & $12(10-15)$ \\
\hline Pyrazinamide & $10(8-12)$ & $15(10-20)$ \\
\hline Ethambutol & $25(20-30)$ & $35(30-40)$ \\
\hline Streptomycin & $15(15-20)$ & $20(15-25)$ \\
\hline
\end{tabular}

Table 3: Recommended doses of first-line anti-tuberculosis drugs for adults $[42,60]$.

There is usually a long delay in diagnosis, due partly to its tendency to mimic other diseases as a result of its varied clinical presentation and radiographic appearance $[3,67,73]$. TB arthritis should be considered in patients who present with indolent symptoms of chronic tenosynovitis [18]. Early diagnosis of arthritis due to TB is essential to preserve the articular cartilage and joint space. The mainstay of treatment is multidrug anti-TB therapy (for 12-18 months) and active-assisted nonweight-bearing exercises of the involved joint throughout the period of healing. Operative intervention (synovectomy and debridement) is required when the patient is not responding after 4-5 months of anti-TB therapy.

\section{References}

1. World Health Organization (2012) Global tuberculosis report 2012. Geneva, Switzerland: WHO.

2. Glaziou P, Falzon D, Floyd K, Raviglione M (2013) Global epidemiology of tuberculosis. Semin Respir Crit Care Med 34: 3-16.

3. Norbis L, Miotto P, Alagna R, Cirillo DM (2013) Tuberculosis: lights and shadows in the current diagnostic landscape. New Microbiol 36: 111-120.

4. Malaviya AN, Kotwal PP (2003) Arthritis associated with tuberculosis. Best Pract Res Clin Rheumatol 17: 319-343.

5. lademarco MF, Castro KG (2003) Epidemiology of tuberculosis. Semin Respir Infect 18: 225-240.

6. Narang S (2012) Tuberculosis of the entheses. Int Orthop 36: 2373-2378.

7. Samuel S, Boopalan PR, Alexander M, Ismavel R, Varghese VD, et al. (2011) Tuberculosis of and around the ankle. J Foot Ankle Surg 50: 466-472.

8. Abdulaziz S, Almoallim H, Ibrahim A, Samannodi M, Shabrawishi M, et al. (2012) Poncet's disease (reactive arthritis associated with tuberculosis): retrospective case series and review of literature. Clin Rheumatol 31: 15211528.

9. Tuli SM (2002) General principles of osteoarticular tuberculosis. Clin Orthop Relat Res: 11-19.

10. Scanzello CR, Goldring SR (2012) The role of synovitis in osteoarthritis pathogenesis. Bone 51: 249-257.

11. Poncet $A$ (1897) De la polyarthrites tuberculeuse deformante ou pseudorheumatisme chronique tuberculeux. Congres Francaise de chirurgerie 1: 732.

12. Sequeira W, Co H, Block JA (2000) Osteoarticular tuberculosis: current diagnosis and treatment. Am J Ther 7: 393-398.

13. Furia JP, Box GG, Lintner DM (1996) Tuberculous arthritis of the knee presenting as a meniscal tear. Am J Orthop (Belle Mead NJ) 25: 138-142.

14. Al-Shaikh R, Goodman SB (2003) Delayed-onset Mycobacterium tuberculosis infection with staphylococcal superinfection after total knee replacement. Am J Orthop (Belle Mead NJ) 32: 302-305. 
15. Varango G, Bamba I, Kodo M, Dao A, Lambin Y (1998) Osteonecrosis of the hip in sickle-cell disease associated with tuberculous arthritis. A review of 15 cases. Int Orthop 22: 384-389.

16. Pointud P, Prudat M, Laluque S, Amouroux J (1993) [Tuberculous arthritis and chondrocalcinosis. Apropos of 2 cases]. Rev Rhum Ed Fr 60: 617-620.

17. Walker GF (1968) Failure of early recognition of skeletal tuberculosis. Br Med J 1: 682-683.

18. Hsiao CH, Cheng A, Huang YT, Liao CH, Hsueh PR (2013) Clinical and pathological characteristics of mycobacterial tenosynovitis and arthritis. Infection 41: 457-464.

19. Chan PC, Chang LY, Wu YC, Lu CY, Kuo HS, et al. (2008) Age-specific cutoffs for the tuberculin skin test to detect latent tuberculosis in BCG-vaccinated children. Int J Tuberc Lung Dis 12: 1401-1406.

20. Wang PD (2009) Assessment of the need for universal BCG vaccination of children in Taipei. Public Health 123: 74-77.

21. Menzies D (2000) What does tuberculin reactivity after bacille CalmetteGuérin vaccination tell us? Clin Infect Dis 3: 71-74.

22. Araujo Z, de Waard JH, de Larrea CF, Borges R, Convit J (2008) The effect of Bacille Calmette-Guérin vaccine on tuberculin reactivity in indigenous children from communities with high prevalence of tuberculosis. Vaccine 26 : 5575-5581.

23. Chen SC, Chen KL, Chen KH, Chien ST, Chen KT (2013) Updated diagnosis and treatment of childhood tuberculosis. World J Pediatr 9: 9-16.

24. Dhanasekaran S, Jenum S, Stavrum R, Wiker HG, Kenneth J, et al. (2014) Effect of non-tuberculous Mycobacteria on host biomarkers potentially relevant for tuberculosis management. PLoS Negl Trop Dis 8: 3243.

25. Arend SM, Thijsen SF, Leyten EM, Bouwman JJ, Franken WP, et al. (2007) Comparison of two interferon-gamma assays and tuberculin skin test for tracing tuberculosis contacts. Am J Respir Crit Care Med 175: 618-627.

26. Ewer K, Deeks J, Alvarez L, Bryant G, Waller S, et al. (2003) Comparison of T-cell-based assay with tuberculin skin test for diagnosis of Mycobacterium tuberculosis infection in a school tuberculosis outbreak. Lancet 361: 11681173.

27. Brock I, Weldingh K, Lillebaek T, Follmann F, Andersen P (2004) Comparison of tuberculin skin test and new specific blood test in tuberculosis contacts. Am J Respir Crit Care Med 170: 65-69.

28. Mori T, Sakatani M, Yamagishi F, Takashima T, Kawabe Y, et al. (2004) Specific detection of tuberculosis infection: an interferon-gamma-based assay using new antigens. Am J Respir Crit Care Med 170: 59-64.

29. Mazurek GH, Villarino ME, CDC (2003) Guidelines for using the QuantiFERON-TB test for diagnosing latent Mycobacterium tuberculosis infection: Centers for Disease Control and Prevention. MMWR Recomm Rep 52: 15-18.

30. Mazurek GH, Jereb J, Lobue P, lademarco MF, Metchock B, et al. (2005) Guidelines for using the QuantiFERON-TB Gold test for detecting Mycobacterium tuberculosis infection, United States. MMWR Recomm Rep 54: 49-55.

31. Detjen AK, Keil T, Roll S, Hauer B, Mauch H, et al. (2007) Interferon-gamma release assays improve the diagnosis of tuberculosis and nontuberculous mycobacterial disease in children in a country with a low incidence of tuberculosis. Clin Infect Dis 45: 322-328.

32. Catanzaro A, Daley C (2013) A summary of the Third Global Interferon-y Release Assay Symposium. Infect Control Hosp Epidemiol 34: 619-624.

33. Pai M, Zwerling A, Menzies D (2008) Systematic review: T-cell-based assays for the diagnosis of latent tuberculosis infection: an update. Ann Intern Med 149: $177-184$.

34. Pai M, Riley LW, Colford JM Jr (2004) Interferon-gamma assays in the immunodiagnosis of tuberculosis: a systematic review. Lancet Infect Dis 4: 761776.
35. Costantino F, de Carvalho Bittencourt M, Rat AC, Loeuille D, Dintinger H (2013) Screening for Latent Tuberculosis Infection in Patients with Chronic Inflammatory Arthritis: Discrepancies Between Tuberculin Skin Test and Interferon-y Release Assay Results. J Rheumatol 40: 1986-93.

36. Perkins MD, Roscigno G, Zumla A (2006) Progress towards improved tuberculosis diagnostics for developing countries. Lancet 367: 942-943.

37. Daniel TM (1988) Antibody and antigen detection for the immunodiagnosis of tuberculosis: why not? What more is needed? Where do we stand today? J Infect Dis 158: 678-680.

38. Gennaro ML (2000) Immunologic diagnosis of tuberculosis. Clin Infect Dis 3: S243-246.

39. Simonney N, Molina JM, Molimard M, Oksenhendler E, Perronne C, et al. (1995) Analysis of the immunological humoral response to Mycobacterium tuberculosis glycolipid antigens (DAT, PGLTb1) for diagnosis of tuberculosis in HIV-seropositive and -seronegative patients. Eur J Clin Microbiol Infect Dis 14: 883-891.

40. Papa F, Cruaud P, David HL (1989) Antigenicity and specificity of selected glycolipid fractions from Mycobacterium tuberculosis. Res Microbiol 140: 569578.

41. Hanza M (1994) Joint and spine tuberculosis. Rev Rheum 60:83-86.

42. Valdazo JP, Perez-Ruiz F, Albarracin A, Sanchez-Nievas G, Perez-Benegas J, et al. (1990) Tuberculous arthritis. Report of a case with multiple joint involvement and periarticular tuberculous abscesses. J Rheumatol 17: 399401.

43. Enarson DA, Fujii M, Nakielna EM, Grzybowski S (1979) Bone and joint tuberculosis: a continuing problem. Can Med Assoc J 120: 139-145.

44. Krama SB, Lee SHS, Abramson SB (2004) Nonvertibral infections of musculoskeletal tuberculosis. In: Rom WN, Garay SM (eds.). Tuberculosis. (2 ${ }^{\text {nd }}$ edn). Lippincott, William \& Wilkins, 577-591.

45. Martini M, Ouahes M (1988) Bone and joint tuberculosis: a review of 652 cases. Orthopedics 11: 861-866.

46. Spiegel DA, Singh GK, Banskota, Ashok K (2005) Tuberculosis of the musculoskeletal system. Tech Orthop 20: 167-178.

47. Wang MN, Chen WM, Lee KS, Chin LS, Lo WH (1999) Tuberculous osteomyelitis in young children. J Pediatr Orthop 19: 151-155.

48. Singh M, Moosa NV, Kumar L, Sharma M (2000) Role of gastric lavage and broncho-alveolar lavage in the bacteriological diagnosis of childhood pulmonary tuberculosis. Indian Pediatr 37: 947-951.

49. Siddiqi S, Ahmed A, Asif S, Behera D, Javaid M, et al. (2012) Direct drug susceptibility testing of Mycobacterium tuberculosis for rapid detection of multidrug resistance using the Bactec MGIT 960 system: a multicenter study. J Clin Microbiol 50: 435-440.

50. den Hertog AL, Daher S, Straetemans M, Scholing M, Anthony RM (2013) No added value of performing Ziehl-Neelsen on auramine-positive samples for tuberculosis diagnostics. Int J Tuberc Lung Dis 17: 1094-1099.

51. World Health Organization (2013) Definitions and reporting framework for tuberculosis - 2013 revision. Geneva, Switzerland.

52. Zhao P, Fang F, Yu Q, Guo J, Zhang JH, et al. (2014) Evaluation of BACTEC MGIT 960 system for testing susceptibility of Mycobacterium tuberculosis to first-line drugs in China. PLoS One 9: e99659.

53. Lawn SD, Mwaba P, Bates M, Piatek A, Alexander H, et al. (2013) Advances in tuberculosis diagnostics: the Xpert MTB/RIF assay and future prospects for a point-of-care test. Lancet Infect Dis 13: 349-361.

54. WHO (2010) Treatment of Tuberculosis: Guidelines $-4^{\text {th }}$ edition. World Health Organization, Geneva, Switzerland.

55. Sawlani V, Chandra T, Mishra RN, Aggarwal A, Jain UK, et al. (2003) MRI features of tuberculosis of peripheral joints. Clin Radiol 58: 755-762. 
56. Gehlot PS, Chaturvedi S, Kashyap R, Singh V (2012) Pott's Spine: Retrospective Analysis of MRI Scans of 70 Cases. J Clin Diagn Res 6: 1534-1538.

57. Facey K, Bradbury I, Laking G, Payne E (2007) Overview of the clinical effectiveness of positron emission tomography imaging in selected cancers. Health Technol Assess 11: iii-iv, xi-267.

58. Papaventsis D, loannidis P, Karabela S, Nikolaou S, Syridou G, et al. (2012) Impact of the Gen-Probe Amplified MTD® Test on tuberculosis diagnosis in children. Int J Tuberc Lung Dis 16: 384-390.

59. Sanjuan-Jimenez R, Morata P, Bermúdez P, Bravo MJ, Colmenero JD (2013) Comparative Clinical Study of Different Multiplex Real Time PCR Strategies for the Simultaneous Differential Diagnosis between Extrapulmonary Tuberculosis and Focal Complications of Brucellosis. PLoS Negl Trop Dis 7: e2593.

60. Kim JH, Kim YJ, Ki CS, Kim JY, Lee NY (2011) Evaluation of Cobas TaqMan MTB PCR for detection of Mycobacterium tuberculosis. J Clin Microbiol 49. 173-176

61. Mattei R, Savarino A, Fabbri M, Moneta S, Tortoli E (2009) Use of the BacT/ Alert MB mycobacterial blood culture system for detection of mycobacteria in sterile body fluids other than blood. J Clin Microbiol 47: 711-714.

62. Chakravorty S, Tyagi JS (2005) Novel multipurpose methodology for detection of mycobacteria in pulmonary and extrapulmonary specimens by smear microscopy, culture, and PCR. J Clin Microbiol 43: 2697-2702.

63. Verettas D, Kazakos C, Dermon A, Petrov H, Galanis V (2003) Polymerase chain reaction for the detection of Mycobacterium tuberculosis in synovial fluid, tissue samples, bone marrow aspirate and peripheral blood. Acta Orthop Belg 69: 396-399.

64. Arthanari S, Yusuf S, Nisar M (2008) Tuberculosis of the knee complicating seronegative arthritis. J Rheumatol 35: 1227-1228.
65. Al-Qattan MM, Al-Namla A, Al-Thunayan A, Al-Omawi M (2011) Tuberculosis of the hand. J Hand Surg Am 36: 1413-1421.

66. Chen SH, Lee $\mathrm{CH}$, Wong T, Feng HS (2013) Long-term retrospective analysis of surgical treatment for irretrievable tuberculosis of the ankle. Foot Ankle Int 34: 372-379.

67. Lawn SD, Zumla Al (2011) Tuberculosis. Lancet 378: 57-72.

68. [No authors listed] (1999) Five-year assessment of controlled trials of shortcourse chemotherapy regimens of 6,9 or 18 months' duration for spinal tuberculosis in patients ambulatory from the start or undergoing radical surgery. Fourteenth report of the Medical Research Council Working Party on Tuberculosis of the Spine. Int Orthop 23: 73-81.

69. Anurag D Bhargava, Anand N Malaviya, Ashok Kumar (1998) Tuberculous rheumatism (poncet's disease)-a case series*. Ind J Tub 45: 215

70. [No authors listed] (1993) Controlled trial of short-course regimens of chemotherapy in ambulatory treatmen of spinal tuberculosis: results at three years of a study in Korea. Twelfth report of the Medical Research Council Working Party on Tuberculosis of the Spine. J Bone Joint Surg Br 75: 240-248.

71. [No authors listed] (1986) A controlled trial of six-month and nine-month regimens of chemotherapy in patients undergoing radical surgery for tuberculosis of the spine in Hong Kong. Tenth report of the Medical Research Council Working Party on Tuberculosis of the Spine. Tubercle 67: 243-259.

72. Pattisson PR (1986) Pott's paraplegia: an account of the treatment of 89 consecutive patients. Paraplegia 24: 77-91.

73. Ellis ME, el-Ramahi KM, al-Dalaan AN (1993) Tuberculosis of periphera joints: a dilemma in diagnosis. Tuber Lung Dis 74: 399-404. 